\title{
Technology and Research
}

\author{
Hannes Devos, PhD \\ Assistant Professor \\ Department of Physical Therapy and Rehabilitation Science, School of Health \\ Professions \\ University of Kansas Medical Center
}

\begin{abstract}
Abiodun Akinwuntan, PhD, MPH, MBA
Dean and Professor, School of Health Professions

University of Kansas Medical Center
\end{abstract}

$\mathrm{T}$

Technology is a word that is frequently used in scientific circles but with no simple definition. It is such a complex word that it has been described to encompass three meanings: tools and instruments; culture; and knowledge. ${ }^{1}$ In the desire to cope with the natural demands of human existence, tools and instruments are developed to solve a variety of problems. These problems, which include but are not limited to transportation, housing, clothing, food cultivation and preservation, nutrition, communication, leisure, entertainment, and health care, are largely driven by human culture. Although the use of technology in changing the world and our immediate environment started around the mid-1700s to 1800s, the current wave of advances in technology really picked up in the mid-1940s. The significant increase in technological advancements around this period was predominantly driven by the advent of computers, new information and communication technologies, and the internet.

The creation of tools and instruments are encoded and passed on from one generation to the other as technological knowledge. For accurate transmission of technological knowledge, research was needed. Research is the systematic investigation and collection of information to establish facts, confirm existing knowledge, or develop new knowledge on any subject matter. As such, empirical observation methods, hypotheses, and inferences on laws concerning behavior of materials and environmental conditions were developed. The effective transmission of knowledge has led to significant advancements in technology, which has continued to revolutionize virtually every aspect of the world we live in today.

The automobile industry is a great example of how technology changed the world. The history of automobiles dates as far back as the $18^{\text {th }}$ century. Since that time, the automobile industry has gone through several technological advancements from steam-powered vehicles, to internal combustion engines, and now electric automobiles. In the early $20^{\text {th }}$ century, Henry Ford introduced the Model $\mathrm{T}$, which was simple and light, yet sturdy enough to drive on the country's primitive roads. The mass production of this automobile lowered its unit price, making it affordable for the middle class, and 
transformed the road network into the driving landscape as we know it.

In the highly motorized world that we live in today, driving has become a primary necessity to the extent that it is regarded as an instrumental activity of daily living. However, driving today is also a highly risky activity with very significant safety implications. The ability to react accurately and promptly to highly time-dependent events is a major requirement to drive safely on public roads. The sequelae of many medical conditions, especially those that affect the nervous and visual systems of the human body, negatively affect the ability to drive. ${ }^{2-7}$ Examples of medical conditions that affect the nervous system include stroke, ${ }^{2}$ multiple sclerosis, ${ }^{3}$ Parkinson's disease, ${ }^{4}$ Alzheimer's disease, ${ }^{5}$ and traumatic brain injury. ${ }^{6}$ Macular degeneration, glaucoma, and retinopathies are some examples of medical conditions that affect the visual system. ${ }^{7}$ The focus of many research endeavors has been to develop the most efficient methods of assessing fitness-todrive of individuals who have experienced any medical condition that has an impact on the ability to drive. 8,9 Furthermore, some research activities have been directed at developing intervention programs that can be used to retrain driving performance after a medical condition. Until very early in the 1980s, assessment and retraining of fitness-to-drive after a medical condition have taken place in real vehicles, on real roads, and in reallife traffic situations. Not only are these practices unsafe, adverse conditions needed to adequately test hazard perception and avoidance skills of the individuals are not guaranteed to happen and are usually avoided, also for safety reasons.
The potential risk of accidents, the lack of standardization and reliability of on-road tests, and the costs associated with onroad driving tests, urged researchers and clinicians to look for safer, cheaper, and more effective technological alternatives to on-road testing.

\section{Driving Simulators}

Driving simulators offer a unique opportunity to conduct fitness-to-drive assessments and interventions in a safe environment. The use of driving simulators for these purposes started about the middle of the $20^{\text {th }}$ century. The driving simulators at that time were primitive and had limited meaningful application in assessing and retraining the full spectrum of skills involved in on-road driving. The early simulators were used predominantly to evaluate the ability of the driver to appropriately control the steering wheel, operate the gas and brake pedals, accurately position the vehicle on the correct road lane, and make simple turns, but without other vehicles on the road. ${ }^{10}$ Performance during more complex driving activities such as driving within the speed limit, overtaking other cars, driving through busy intersections, and making left turns against oncoming traffic could not be assessed nor retrained in the early simulators. However, technological advancements have led to the development of high fidelity virtual reality driving simulators in which the more complex driving activities as well as the simple ones can be assessed and retrained with a high degree of ecological accuracy. ${ }^{11}$ Simulator-based assessment and intervention now offer researchers and driving assessment experts the opportunity to evaluate and retrain driving be- 
haviors in near-realistic driving situations that elicit natural reaction to such situations. For example, a driver's response to a potentially dangerous maneuver by another driver or driving performance during adverse weather or lighting conditions can be readily evaluated in current driving simulators. The fact that the simulated drives are performed without the risk inherent in reallife driving situations and can be recorded and replayed at a future time enhance the utility of driving simulators as innovative tools for evaluating and retraining driving performance, especially in persons with medical conditions.

In various studies, we established the usefulness of a driving simulator to retrain impaired driving skills after different medical conditions including stroke, multiple sclerosis, and Parkinson's disease. ${ }^{11-14}$ In the stroke study, a total of 83 participants between six and nine weeks after the onset of a first-ever stroke were recruited into a randomized controlled trial. ${ }^{11}$ Forty-two of them were randomly allocated to an experimental group that received 15 hours of structured training in a high fidelity driving simulator. All simulator scenarios were specifically created for this study and tailored to the needs of each individual driver. The other 41 participants were allocated to a control group that received 15 hours of structured cognitive rehabilitation using off-the-shelf puzzle games. In both groups, the 15 hours of training, which took place in one hour sessions, three times a week over five weeks, was in addition to standard rehabilitation. Before commencement and immediately after training, all participants performed a comprehensive fitness-to-drive evaluation that included assessments of visual, cognitive, and practical on-road driving skills. Performance on the same tests that were conducted as part of an official state-mandated fitness-to-drive evaluation at about six months after stroke onset was also documented. The study results showed improvements on the visual, cognitive, and on-road tests immediately after training in both groups and the benefit in the experimental group was superior. At the six-month evaluation, significantly $(p=0.03)$ more participants in the experimental group $(73 \%)$ in comparison to the control group (42\%) passed the fitness-to-drive after evaluation and were legally allowed to resume driving. In a long-term follow-up study that was conducted at about five years after the stroke, $60 \%$ of the 30 experimental group participants in comparison to $48 \%$ of the 31 controls again passed the official statemandated fitness-to-drive evaluation. ${ }^{12}$ Similar benefits of using the high fidelity driving simulator to improve performance of driving-related skills were observed in others studies that we conducted that included individuals with relapsing remitting multiple sclerosis ${ }^{13}$ and persons with mild to moderate physical disabilities due to Parkinson's disease. ${ }^{14}$

Although the interest in driving simulation technology is growing, clinicians are still apprehensive of using simulators to evaluate and train driving skills of patients with medical conditions. Concerted efforts should be made by researchers and software manufacturers to facilitate the transition of research technology to practical implementation in the clinical community. Examples include creating user-friendly interfaces to ease 
the operation of the simulator, providing summary print-outs of the data that can easily be interpreted, and demonstrating the validity of simulation technology to real-world driving performance.

\section{Future Plan}

In future studies, we plan to look at the benefits of measuring cognitive workload while performing different cognitive tasks of varying levels of difficulty in the driving simulator. Cognitive workload is defined as the mental effort needed to execute a task. ${ }^{15}$ For decades, the only established methods of measuring cognitive workload were positron emission tomography or functional magnetic resonance imaging. These neuro-imaging measures were sometimes intrusive and required subjects to lie still in a scanner while taking pictures of the brain. Consequently, these scanning techniques are not optimal to study complex cognitive tasks while driving. Recent advancements in technology showed that other measures, including electroencephalography or magneto-encephalography, functional near-infrared spectroscopy , cardiovascular measures (blood pressure), electrodermal measures, and pupillometry (changes in pupil size), can be used outside the scanner to assess cognitive workload. ${ }^{15}$ In our future studies, we will use pupillometry that is evoked by specific cognitive tasks with established levels of complexities because it accurately reflects fluctuations in cognitive activity over the entire time of engaging in the task. Taskevoked pupillary response (TEPR) is an involuntary reflex, caused by a decrease in parasympathetic activity in the peripheral nervous system as a result of increased cognitive workload. ${ }^{16}$ Our main hypothesis, based on findings in other studies, is that the known differences in cognitive workload between individuals with diagnosed progressive neurological conditions when compared to healthy individuals matched for age and education will be more accurately detected using pupillary size changes. Accurate detection of abnormal changes in cognitive workload may be a precursor and earliest indicator of cognitive decline in progressive neurological conditions. Early detection of cognitive decline will inform targeted interventions capable of slowing down the rate of disease progression thereby prolonging highest quality of life. It is our hope that as our studies develop further, we will attract the right partnership within the industry to develop innovative products that are very sensitive in detecting earliest onset of cognitive decline and offer the possibility of earlier introduction of effective interventions.

Advocacy for Collaborative Research

For advancements to continue to have meaningful and translational impacts on all the aspects of human activities that are technology-influenced, transmission of technological knowledge to healthcare and industry will need to be more effective and accurate. More randomized controlled trials and long term follow up studies are needed to ensure proper transmission of advancements in technology. Such trials should include reliable and valid measures to enable reproducibility and generalization from one research location to another. Associated with the increasing complexities of technology, is the issue of new forms and huge amounts of data generated by the technologies. Data mining and management are therefore important skills to 
possess or procure in conducting research around current technology. Fortunately, advancements in computer technology have made effective handling, storage, and accurate analyses of humongous quantitative and qualitative data possible. Knowledge gained from technologically-based research, like any other research, should be comprehensively documented, disseminated widely, and published in peer-reviewed journals in order to add to the body of knowledge.

To keep the vehicle of technological advancements going, it is important for universities and the industry to continue to engage in scientific partnerships. For a successful university-industry partnership, not only is the support and belief of the leadership of both organizations in

\section{References}

1. Vergragt PJ. How technology could contribute to a sustainable world.

Tellus Institute 2006. 1-29.

2. Devos H, Akinwuntan AE, Nieuwboer A, Truijen S, De Weerdt W. A systematic review and meta-analysis on predictors of fitness to drive after stroke. Neurology 2011; 76(8): 74756.

3. Lincoln NB, Radford KA. Cognitive abilities as predictors of safety to drive in people with multiple sclerosis: Multiple Sclerosis 2008; 14(1): 123-128.

4. Devos H, Vandenberghe $W$, Nieuwboer A, Tant M, Baten G, De Weerdt $W$. Predictors of fitness to drive in people with Parkinson disease. Neurology 2007; 69 (14): 1434-41. the partnership crucial, it needs to be a top priority. The appropriate manpower, resources, and incentives needed to ensure the success of the partnership need to be available in both organizations. Such partnerships should not be bogged down by the bureaucracies and selfish interests of both organizations. As such, it is important for a detailed memorandum of understanding that clearly establishes all the terms of agreement to ensure effective communication and clearly defined expectations between the parties in the partnership to be developed. According to Edmondson et al, 2012, when such partnerships work well, they merge the discovery-driven culture of the university with the innovation-driven environment of companies. ${ }^{17}$

5. Ranchet $\mathrm{M}$, Tant $\mathrm{M}$, Akinwuntan AE, Morgan JC, Devos H. Fitness-todrive disagreements in individuals with dementia. The Gerontologist 2016, August 5 (Epub ahead of print).

6. Schultheis MT, Whipple E. Driving after traumatic brain injury: evaluation and rehabilitation interventions. Current Physical Medicine and Rehabilitation Reports 2014; 2(3): 176183.

7. Klein R. Age-related eye disease, visual impairment, and driving in the elderly. Human Factors 1991; 33(5): 521-525.

8. Devos H, Ranchet M, Akinwuntan AE, Uc EY. Establishing an evidencebased framework for driving rehabilitation in Parkinson's disease: a systematic review of on-road studies. 
NeuroRehabilitation 2015; 37(1): 3552.

9. Devos H, Akinwuntan AE, Gélinas I, George S, Nieuwboer A, Verheyden

G. Shifting up a gear: considerations on assessment and rehabilitation of driving in neurological conditions. Physiotherapy Research International 2012. 2012; 17(3): 125-31.

10. Kent H, Sheridan J, Wasko E, June C. A driver training program for the disabled. Archives of Physical Medicine and Rehabilitation 1979; 60: 273276.

11. Akinwuntan AE, De Weerdt $W$, Feys H, Baten G, Arno P, Strypstein E, Kiekens C. Effect of simulator training on the driving ability of stroke patients: a randomized controlled trial (RCT). Neurology 2005; 65: 843850.

12. Devos H, Akinwuntan AE, Nieuwboer A, Tant M, Truijen S, De Wit L, Kiekens C, De Weerdt W. Effect of simulator training on driving after stroke: a 5 year follow-up of a randomized trial. Neurorehabilitation and Neural Repair 2010; 24(9): 843850.

13. Akinwuntan AE, Devos H, Baker K, Hoogstra M, Phillips K, Kumar V, Smith S, Williams JM. Improvement of driving skills in persons with Relapsing Remitting Multiple Sclerosis: a pilot study. Archives of Physical Medicine and Rehabilitation 2014; 95(3): 531-537.
14. Devos H, Morgan JC, Onyeamaechi A, Bogle CA, Holton K, Kruse J, Sasser S, Akinwuntan AE. The use of a driving simulator to improve onroad driving performance and cognition in persons with Parkinson's disease: a pilot study. Australian Occupational Therapy Journal 2016, April 13 (Epub ahead of print).

15. Ranchet M, Morgan JC, Akinwuntan $\mathrm{AE}$, Devos H. Cognitive workload in healthy aging and age-related neurodegenerative diseases: A systematic review of physiological measures. In-Review.

16. Beatty J. Task-evoked pupillary responses, processing load, and the structure of processing resources. Psychological Bulletin. 1982; 91: 276292.

17. Edmondson G, Valigra L, Kenward M, Hudson RL, Belfield H. IndustryUniversity Partnerships Work: Lessons from successful collaborations. Business Innovation Board AISBL of the Science, 2012. 1-50. 